\title{
Transfer Robustness Optimization for Urban Rail Transit Timetables
}

\author{
Liqiao Ning (D), Peng Zhao (D), Wenkai Xu (D), and Ke Qiao \\ School of Traffic and Transportation, Beijing Jiaotong University, Beijing, China \\ Correspondence should be addressed to Peng Zhao; bjtu_zhaopeng@163.com
}

Received 21 April 2018; Revised 31 August 2018; Accepted 17 September 2018; Published 1 October 2018

Academic Editor: Seungjae Lee

Copyright (C) 2018 Liqiao Ning et al. This is an open access article distributed under the Creative Commons Attribution License, which permits unrestricted use, distribution, and reproduction in any medium, provided the original work is properly cited.

\begin{abstract}
A good timetable is required to not only be efficient, but also yield effectiveness in preventing and counteracting delays. When travelling via urban rail transit networks, transferring passengers may miss their scheduled connecting train because of a feeder train delay that results in them experiencing increased travel costs. Considering that running time supplements and transfer buffer times yield different effects on the travel plans of transferring and nontransferring passengers, we formulate an expected extra travel cost (EETC) function to appropriately balance efficiency and robustness, which is then implemented in the construction of a robust transfer optimization model with the objective of minimizing the total EETC. Next, to improve the computational efficiency, we propose an approximate linearization approach for the EETC function and introduce two types of binary variables and auxiliary substitution variables to convert the nonlinear model to a mixed-integer linear model. Experimental results show that our proposed method can yield practically applicable solutions with significant reductions in both EETC and probability of missing a transfer.
\end{abstract}

\section{Introduction}

Concurrently optimizing train timetables and enhancing robustness is the main problem on the operational level of railway systems; in general, optimization aims to prevent and counteract delays such that the deviations of the original timetable are as small as possible. Currently, there are four general methods to improve the robustness of train timetables: (1) adding recovery time, (2) decreasing capacity for a given traffic pattern, (3) decreasing heterogeneity, and (4) decreasing average speed [1]. For an urban rail transit system with fixed capacity, the most widely used method is adding recovery time that includes a running time supplement and dwell time supplement, as shown in Figure 1. The standard running time is the ideal running time required for a specific train to travel from one station to another. The running time supplement is primarily utilized to offset train delays occurring during operation. The standard dwell time is the dwell time necessary for passengers to board and alight. The dwell time supplement is primarily purposed to manage fluctuations in passenger volume at stations and to ensure that trains depart on schedule. Besides, the headway buffer between two consecutive trains, the difference between the headway and minimum headway, enables the second train to run at unrestricted speed, which has a direct influence on the capacity of urban rail transit system. Landex [2] held that the more trains operated on a given railway line, the higher the risk to encounter delays. The capacity issue is a systematic and complex problem on the crowded urban rail line.

Because trains are operated within a network, a single train delay can affect other lines via transfers; consequently, this delay may postpone the departure of scheduled connections. Missing a transfer is a crucial measure in the evaluation of network timetable performance, as has been mentioned in many research studies, such as Dollevoet [3], Corman, D’Ariano, and Hansen [4], and Parbo, Nielsen, and Prato [5]. Furthermore, because the headway between trains on suburban rail transit lines is typically longer for the majority of the day, missing a transfer implies a long mean waiting time for transferring passengers that significantly affects the level of service. Therefore, with the expansion of suburban rail transit networks in many large cities, it has become essential to optimize the robustness of transfer timetables in order to provide high-level service and obtain satisfaction with operations.

A running time supplement and transfer buffer time can each yield improvements in the robustness of the train 


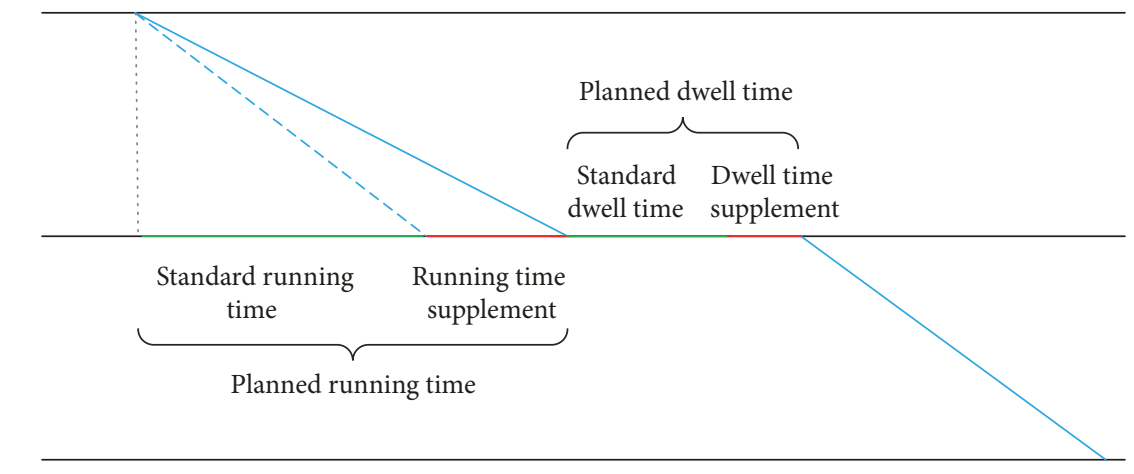

FIGURE 1: Adding recovery time method illustrating running time and dwell time supplements.

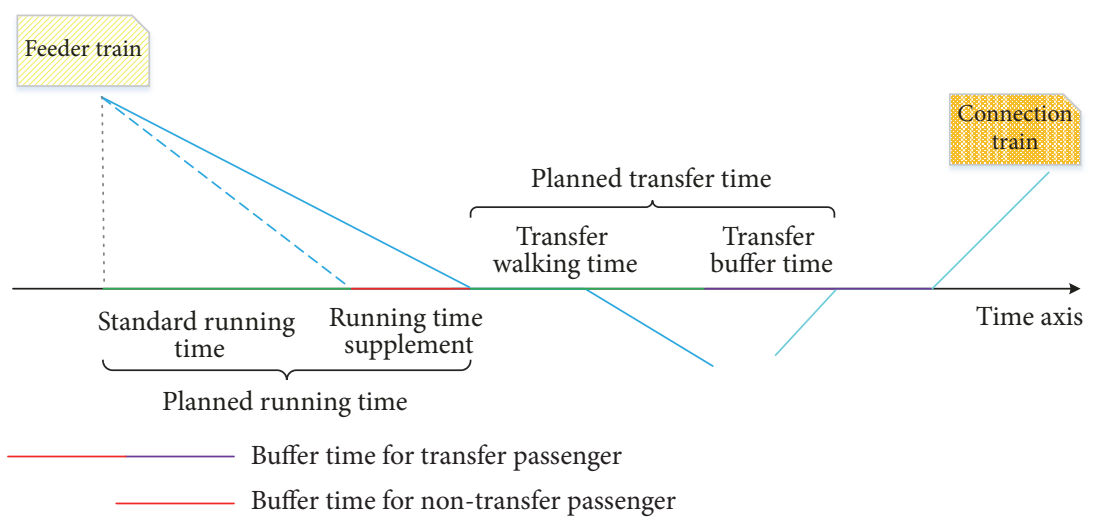

FIgURE 2: Double-buffer effect.

timetabling problem with respect to transfer connections. However, in terms of a connection, for nontransferring passengers, their travels are only affected by the running time supplement while the travels of transferring passengers are affected by both the running time supplement and transfer buffer time, a phenomenon that is herein referred to as the "double-buffer effect." The double-buffer effect is illustrated in Figure 2. Most researchers are dedicated to optimizing timetable robustness by adjusting only the running time supplement. It seems unfair for nontransferring passengers because they potentially need to wait more time that is in effect the buffer for transferring passengers. However, transfer buffer time is specially used to buffer for transferring passengers and would not hold up the travels of nontransferring passengers. Alternatively, based on the notion of the double-buffer effect, the first contribution of this study is optimizing timetable robustness via simultaneous adjustment of the running time supplement and transfer buffer time (we do not consider the dwell time supplement addressing the demand fluctuations at station in this paper). Accordingly, depending on the value of time (VOT) and distribution of train delays, an expected extra travel cost (EETC) function of all passengers (transferring, passing, departing, and arriving passengers) can be formulated as a nonlinear function of the running time supplement and transfer buffer time.

The EETC is a probabilistic weighted function of all cases of delay to occur that incorporates the cases of successful and missed transfers with respect to transferring passengers and the cases of early-arriving and late-arriving with respect to nontransferring passengers. It is clear that both the efficiency and robustness have an effect on EETC in practice. Therefore, a robust transfer optimization model is subsequently developed by minimizing the total EETC throughout a network, which is the second contribution of this study. Implementing the total EETC yields the following three advantages: (1) a more accurate description of the effects of buffer time, (2) an appropriate compromise of efficiency and robustness to avoid too small or too large buffer times, and (3) a coordination of all of the relevant buffer times throughout a network as a whole.

However the nonlinearization of the objective function makes solving the optimized model complex and difficult. Hence, the third contribution of this study is putting forward an improved approximate linearization approach that, respectively, linearizes the EETC function for transferring and nontransferring passengers. In addition, a further linearization of the objective via introduction of binary variables and auxiliary substitution variables is proposed to entirely convert the optimized model to a mixed integer linear programming model. The improved approach takes into account both the difference between the effects of buffer time on transferring and nontransferring passengers and the fact that the running time supplement and transfer buffer time yield equivalent effects on transferring passengers. Finally, 
an experimental analysis is conducted to demonstrate the performance of our proposed methodology.

The remainder of this paper is organized as follows: Section 2 provides literature review; Section 3 provides the EETC function formulation; Section 4 establishes a robust transfer optimization model; Section 5 proposes an approximate linearization approach with respect to the characteristics of local EETC and demonstrates the secondary linearization; Section 6 provides experimental analyses; and Section 7 provides the conclusions and future research directions.

\section{Literature Review}

Generally, optimization of train timetabling can be performed from the perspective of robust train timetabling or nominal train timetabling. However, in many cases, the latter, and most efficient, solution yields worse performance in actual operation [6]. For example, although synchronization timetabling aims to generate timetable solutions to reduce passenger transfer waiting time as much as possible, in the case of synchronization, once a delay occurs, transferring passengers will miss their scheduled connecting train. In fact, transfer waiting time can be used as a buffer in robust level. When required to choose one among several efficient solutions, operators prefer to implement a more robust solution rather than the most efficient solution. Furthermore, a discrepancy exists between how train-oriented operations are planned with the main focus being on the trains and how passengers actually perceive and respond to railway performances. Hence, researches on robust train timetabling can be conducted with the train-oriented and passengeroriented focus [5].

2.1. Train-Oriented Robust Train Timetabling. Traditionally, the focus in robust train timetabling is train-oriented, aiming to ensure the on-time performance of the trains. Kroon et al. [7] proposed a two-stage stochastic programming approach to minimize the average weighted delay of all trains by allocating a time supplement and buffer time. Jovanović et al. [8] modelled the allocation of slack times as a knapsack problem, where the profit for inserting a buffer corresponded to the importance of reducing delay propagation. Alternatively, Fischetti and Monaci [9] introduced the concept of light robustness by allowing deterioration of the nominal timetable objective function value. A set of slack variables was used to measure the robustness of solution, and their sum was minimized in the objective function. D'Ariano, Pacciarelli, and Pranzo [10] referred to the flexible timetable in which fewer details are fixed in schedule and dynamic traffic management was herein to solve the conflicts during operations, and it allowed more freedom to the real-time control. Liebchen et al. [11] presented the concept of recoverable robustness that a solution was recovery robust if it could be recovered by limited means in all likely scenarios. D'Angelo et al. [12] and Goerigk and Schöbel [13] also applied this concept of robustness. Besides, when very frequently delays arise, the delay management should be taken into account at an operational level. In consideration of the efficiency in real-time rescheduling, Samà et al. [14] were dedicated to reducing the related train delays and developing efficient algorithms to fast rescheduling trains. Andersson, Peterson, and Krasemann [15] presented an optimization approach to reduce the propagation of delays by introducing a more efficient margin time allocation in the timetable.

The above research mainly focused on the robustness, and the efficiency of timetable was generally addressed as a constraint on the maximum efficiency loss; or the robustness optimization aimed at enhancing the ability to absorb minor disruptions and the recoverability of the schedule; however, they would get trouble in describing the size of "minor" delays. Some researches were conducted with the motivation of finding robust yet efficient solutions. Schlechte and Borndörfer [16] proposed a bicriteria optimization approach by considering the robustness and efficiency to be incomparable entities. Cacchiani, Caprara, and Fischetti [17] approximately solved a relaxation of the timetable through an iterative Lagrangian optimization scheme capable of dealing with robustness. During the optimization process, a set of, roughly speaking, "Pareto optimal" heuristic solutions could be collected. Lee et al. [18] developed a simulation-based timetable robustness and efficiency heuristic approach by changing the penalization threshold for the total time supplement. Generally, the efficiency depended on the amount of time supplement, and the robustness was related to the unscheduled delay. Although these studies provided the Pareto optimal solutions to numerically establish the relationship between robustness and efficiency, they still failed to find a compromise and balance between the robustness and efficiency, because the train-related measurements are of different dimensions for the robustness and efficiency. Some researchers introduced the weighted value for the different objective. However, it is too arbitrary and subjective in this way.

2.2. Passenger-Oriented Robust Train Timetabling. Without knowing or monitoring the exact impact on the passengers' travel experience, train-oriented robust train timetabling would not take the passengers explicitly into account. However, the optimal timetabling for the operators could be far from optimal for the passengers with the reasons being cancelled trains, missed transfers, or route choice adaptations [19]. Recent studies have emphasized that exhaustive passenger-oriented measurements are needed, and maintaining transfers is often the main concern.

Many researchers conducted delay management studies that required operators to make a decision on whether or not to keep a connection when trains were delayed. In this regard, Kanai [20] combined the simulation and optimization to minimize passengers' dissatisfaction. The decision of keeping or dropping connections was solved by a tabu search algorithm and evaluated by a passenger simulation model. Corman et al. [21] developed a biobjective delay management strategy to minimize the number of missed connections and avoid train conflicts when rescheduling. Nesheli and Ceder [22] took holding, skip-stops, and short-turn control statics into account to minimize total passenger travel time and maximize direct (without waiting) transfers real-time control action. 
The delay management problem is for a fixed plan and a fixed scenario of delays, while the delay resistant timetabling optimizes with respect to all scenarios of delays [23]. Liebchen and Stiller [24] optimized the allocation of a certain buffer budget to achieve the maximum resistance against delays, which simplified the distributions of delay on each arc to two values, a fixed delay with a certain probability $p$ and no delay with the probability 1- $p$. In this case, the whole system had billions of different delay scenarios. Schöbel and Kratz [25] established a bicriteria model built on a set of determined delays and proposed a Pareto optimization technique for the balance of the efficiency and robustness. The timetable robustness was defined as the largest possible passenger delay such that all transfers were maintained under some given strategy. Liebchen et al. [23] modelled the periodic event-activity networks with an expansion of a driving and waiting activity to (three) different scenarios where the weight of transfer arcs was relative to probabilities of the scenarios. Even though the above research attempted to allow for all scenarios of delays, the delay distribution was in fact assumed as discretized, finite, and determined beforehand, and the optimized objective was weighted by a set of scenarios where the delay of driving and waiting activities had several possibilities. Alternatively, Vansteenwegen and Van Oudheusden $[26,27]$ introduced an expected passenger waiting time function of running time supplement as the objective to weigh total minutes spent waiting across all passengers for purpose of the appropriate allocation of running time supplement, in which the transfer buffer time was assumed to be zero. The function emphasized the effects of missed transfer by setting a larger value of time. Dewilde [28] and Sels et al. [29] also applied this concept of robustness, which allowed for all cases of delay. However, the researches failed to recognize the effects of transfer buffer time and the double-buffer effect between running time supplement and transfer buffer time. Conversely, Goverde [30] focused on the effects of the transfer buffer time to formulate the expected waiting time for transferring passengers while omitting the effects of the running time supplement.

In summary, the previous research in the field of robust train timetabling is typically done by allocating the time supplements. Even when it comes to reducing the risk of missing transfer connections, the effects of transfer buffer time are often overlooked. Recent researches attempt to cope with the issues but are too rough. The delay distributions are assumed as several possibilities and determined beforehand in regard to the practicability of model. Alternatively, the EETC is weighted by the probabilities of all cases of delay and can be embedded in many robustness researches. To the best of our knowledge, our paper is the first attempt to explicitly describe and distinguish the effects of the running time supplement and transfer buffer time to formulate an EETC. To optimize one of them or regard them as single combined buffer times would make the measured objective unfair when applied to all connections within a network in terms of the double-buffer effect. The travels of nontransferring passengers only necessitate consideration of the running time supplement and are thus exclusive of transfer buffer time. Based on the difference, an improved approximate linearization approach is proposed.

\section{Expected Extra Travel Cost (EETC) Function}

Weston et al. [31] held that, because of missed transfers, minimizing train delay did not necessarily minimize passenger delays. Therefore, from the perspective of passengers, a guarantee of reliable transfers is crucial for urban rail transit systems. In this paper, we formulate the expected extra travel cost (EETC) function to explicitly describe the passengers' perception of trip.

3.1. Distribution of Train Delay. Note that the train delay time is defined as the difference between actual running time and standard running time in this paper. Generally, the negative exponential distribution has often been used to model the train primary delay in the literature $[26,27,29$, 32]. Moreover, several statistical analyses of empirical data have also confirmed this hypothesis [33-35]. The probability density function and distribution function can be expressed as

$$
\begin{aligned}
& f(x)=\lambda e^{-\lambda x} \\
& F(x)=1-e^{-\lambda x}
\end{aligned}
$$

where $x$ is the primary delay time and $1 / \lambda$ is the mean delay time.

Here, we denote the train delay time as $d$, the running time supplement as $S$, the transfer buffer time as $B$, and the headway between trains on connection line as $h$. Three distinct cases of actual running time are defined in accordance with the respective time horizon, as illustrated in Figure 3.

Case $1(d \leq S)$. In this case, the connecting train should remain at the station for a period of time $S-d$ to depart at the scheduled departure time.

Case $2(S<d \leq S+B)$. In this case, the transfer buffer time affords transferring passengers time to catch their scheduled connecting train.

Case $3(d>S+B)$. In this case, because the delay time exceeds the running time supplement and the buffer time, transferring passengers will miss the scheduled connection and must wait for the next connecting trains.

From the perspective of train operation, Case 1 is the early-arriving circumstance, and Cases 2 and 3 are the latearriving circumstances, which result in scheduled delays. However, in terms of transfers, Cases 1 and 2 result in successful transfers, while Case 3 results in a missed transfer.

3.2. Expected Extra Travel Cost Function. Passengers at a single station are categorized as follows according to their type of travel relative to the station: transferring, passing, departing, and arriving passengers. The EETC function for passengers of each type is a probabilistic weighted value depending on 


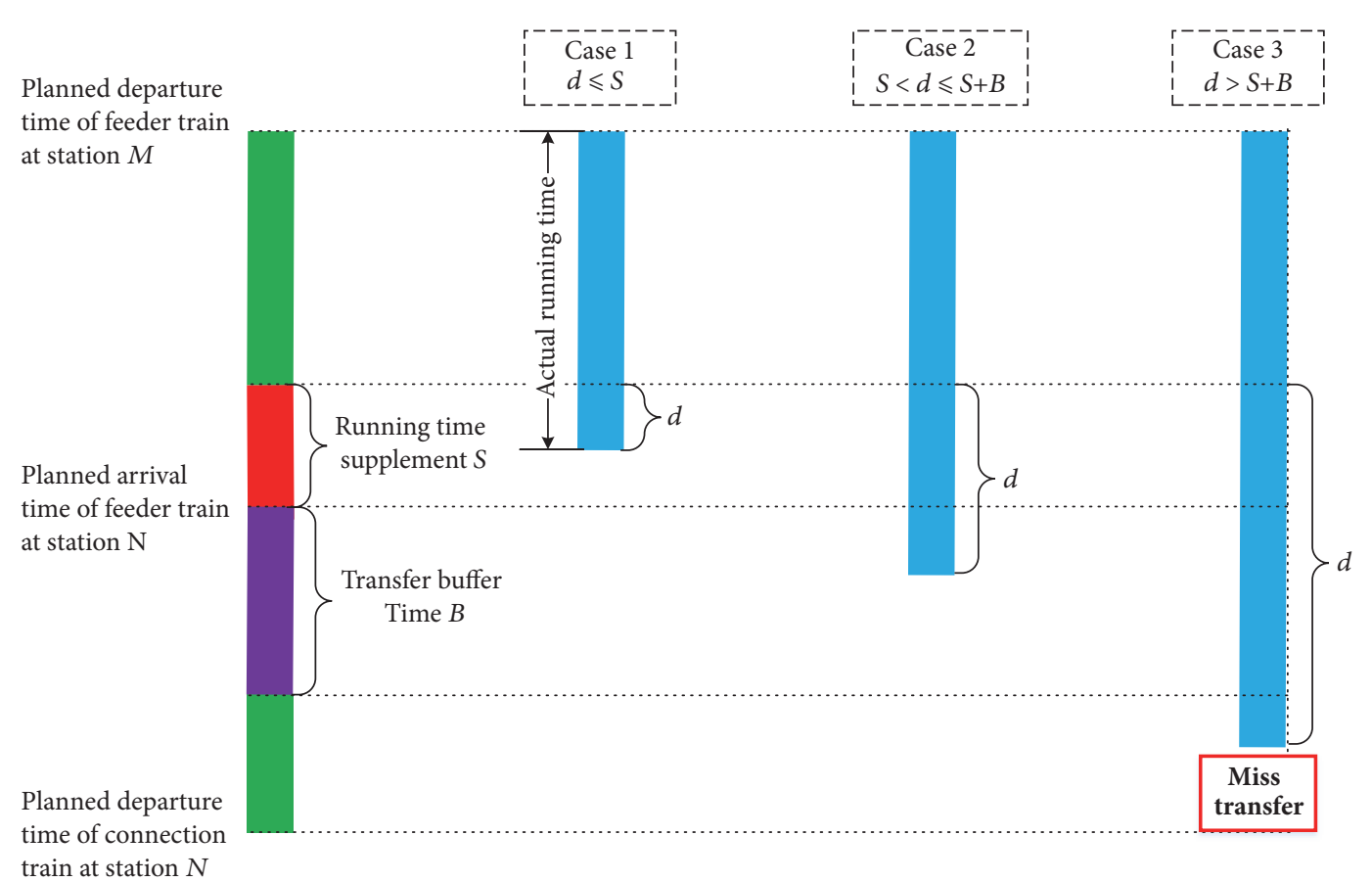

FIgURE 3: Three types of actual running time.

the VOT and distribution of train delays in practice. In this paper, the standard travel time equals standard in-vehicle time that includes standard running time and standard dwell time. The extra travel time includes the extra in-vehicle time (the difference between actual and standard in-vehicle time) and the extra waiting time on platform. A substantial amount of research has been carried out to determine the value of time (VOT) in various circumstances [36-39]. Given that each passenger has different feelings concerning the scheduled and unscheduled travel time, we refer to these research results and, respectively, set the VOT for cases of scheduled and unscheduled, as indicated in Table 1.

Here, we define the VOT for the standard in-vehicle time as equal to one. The VOT for the scheduled extra in-vehicle time is equal to 1.5 , which means that passengers value 1 min. of the scheduled extra in-vehicle time as $1.5 \mathrm{~min}$. of the standard in-vehicle time. As an unscheduled extra waiting yields undesirable effects on passenger travel, the VOT for the unscheduled extra in-vehicle time is set to 2.0. The VOT for the scheduled and unscheduled waiting time are similarly set to 2.0 and 2.5, respectively. Because missing a transfer yields more significant adverse effects, the VOT for the waiting time due to a missed transfer is 2.7 , which is a value greater than those previously mentioned. This increased VOT is justified by the extended waiting time that would be attributed to the long headway between connections.

3.2.1. Transferring Passenger. The total buffer time for a transferring passenger is the sum of the running time supplement and transfer buffer time. If delay time $d$ is less than the total buffer time $S+B$, transferring passengers are able to board the scheduled connecting train, which departs according to schedule. In this case, the extra travel time utilized by transferring passengers consists of the scheduled extra invehicle time $d$ and the scheduled extra waiting time $S+B-$ $d$. Then, the EETC for each transferring passenger can be calculated as

$$
\begin{aligned}
& C_{t r}^{w}=\int_{0}^{S+B}\left(V_{1} d+V_{2}(S+B-d)\right) \lambda e^{-\lambda d} d d \\
& =\frac{V_{1}-V_{2}+\lambda V_{2}(S+B)+\left(V_{2}-V_{1}-\lambda V_{1}(S+B)\right) e^{-\lambda(S+B)}}{\lambda}
\end{aligned}
$$

Conversely, when delay time $d$ is greater than the total buffer time $S+B$, transferring passengers miss the scheduled connection and must wait for the next connecting train. Thus, in this case, transferring passenger extra travel time comprises the scheduled extra in-vehicle time $S+B$, the unscheduled extra in-vehicle time $d-(S+B)$, and the waiting time because of the missed transfer $h-(d-S-B)$. Then, the EETC for each transferring passenger can be calculated as

$$
\begin{aligned}
C_{t r}^{m} & =\int_{S+B}^{+\infty}\left(V_{1}(S+B)+V_{3}(d-S-B)\right. \\
& \left.+V_{5}(h-d+S+B)\right) \lambda e^{-\lambda d} d d \\
& =\frac{\lambda V_{1}(S+B)+\lambda V_{5} h+V_{3}-V_{5}}{\lambda} e^{-\lambda(S+B)}
\end{aligned}
$$

3.2.2. Passing Passenger. The trips made by passing passengers only necessitate consideration of the running time supplement and are thus exclusive for transfer buffer time. When delay time $d$ is less than the running time supplement $S$ and the feeder train departs according to the scheduled 
TABLE 1: VOT for different circumstances.

\begin{tabular}{cccccc}
\hline \multirow{2}{*}{ Cases } & \multicolumn{2}{c}{ Scheduled } & \multicolumn{2}{c}{ Unscheduled } \\
& In-vehicle time $V_{1}$ & Waiting time $V_{2}$ & In-vehicle time $V_{3}$ & Waiting time $V_{4}$ & Waiting time due to a missed transfer $V_{5}$ \\
\hline VOT & 1.5 & 2.0 & 2.0 & 2.5 & 2.7 \\
\hline
\end{tabular}

timetable, the extra travel time of passing passengers is always equal to the running time supplement $S$. Then, the EETC for each passing passenger can be calculated as

$$
C_{t h}^{e}=\int_{0}^{S} V_{1} S \lambda e^{-\lambda d} d d=V_{1} S-V_{1} S e^{-\lambda S}
$$

Accordingly, when the delay time $d$ is greater than the running time supplement $S$, the extra travel time of passing passengers comprises the scheduled extra in-vehicle time $S$ and the unscheduled extra in-vehicle time $d-S$. Then, the EETC for each passing passenger can be calculated as

$$
\begin{aligned}
C_{t h}^{l} & =\int_{S}^{+\infty}\left(V_{1} S+V_{3}(d-S)\right) \lambda e^{-\lambda d} d d \\
& =\frac{\left(\lambda V_{1} S+V_{3}\right) e^{-\lambda S}}{\lambda}
\end{aligned}
$$

3.2.3. Arriving Passenger. For arriving passengers, when the delay time $d$ is less than the running time supplement $S$, the extra travel time is equal to the delay time $d$. Thus, the EETC for each arriving passenger can be calculated as

$$
C_{a r}^{e}=\int_{0}^{S} V_{1} d \lambda e^{-\lambda_{\eta} d} d d=\frac{V_{1}-\left(\lambda V_{1} S+V_{1}\right) e^{-\lambda S}}{\lambda}
$$

Additionally, in the case of a late-arriving train, the extra time of arriving passengers is equivalent to that of passing passengers; the EETC in this case can be calculated as

$$
C_{a r}^{l}=\frac{\left(\lambda_{\eta} V_{1} S+V_{3}\right) e^{-\lambda S}}{\lambda}
$$

Besides, in urban rail transit network, there are some specific stations that are accessed by feeder bus, and the arriving passengers can transfer to bus network to go to their destination. In this case, the EETC is supposed to take into account the feeder bus timetable. In terms of transfers involving bus transit lines, passengers are also likely to encounter a missed transfer, thus, and there are two circumstances: catching and missing the scheduled feeder bus. The calculation of EETC can be implemented similar to transferring passengers.

3.2.4. Departing Passenger. In the case of an early arrival, as the feeder train departs according to the scheduled timetable, there is no extra travel time for departing passengers; therefore, $C_{d e}^{e}=0$. However, if delay time $d$ is greater than the running time supplement $S$, then the extra travel time of departing passengers equates to the unscheduled extra waiting time $d-S$. Accordingly, the EETC for each departing passenger can be calculated as

$$
C_{d e}^{l}=\int_{S}^{+\infty} V_{4}(d-S) \times \lambda e^{-\lambda d} d d=\frac{V_{4} e^{-\lambda S}}{\lambda}
$$

3.2.5. Expected Extra Travel Cost. The EETC is the expected extra travel cost of all cases of delay with the specific probability distribution. For a connection, the local EETC of passengers is the weighted sum of the EETC of all types of passengers, (3)-(9), as expressed via (10). The local EETC is a nonlinear and probability function of the running time supplement $S$ and transfer buffer time $B$ corresponding to the connection and implies the probability distributions of delay, which is expressed as follows:

$$
\begin{aligned}
C(S, B) & \\
= & P_{t r} \frac{-0.5+2 \lambda(S+B)+(-0.2+2.7 \lambda h) e^{-\lambda(S+B)}}{\lambda} \\
& +P_{t h} \frac{1.5 \lambda S+2 e^{-\lambda S}}{\lambda}+P_{a r} \frac{1.5+0.5 e^{-\lambda S}}{\lambda} \\
& +P_{d e} \frac{2.5 e^{-\lambda S}}{\lambda}
\end{aligned}
$$

where $P_{t r}, P_{t h}, P_{a r}$, and $P_{d e}$ represent the numbers of transferring, passing, arriving, and departing passengers, respectively.

\section{Robust Transfer Optimization Model}

Ideally, passengers without any risk-preference expect to minimize their transfer waiting time. However, in case of short transfer time, missing a transfer may occur due to train delays. The focus of robust transfer optimization is to guarantee the transfer reliability at a certain efficient level of transferring. In more detailed ways, the concept of robustness requires the compromise and balance of the efficiency and robustness; in this case, the buffer times should be large enough to compensate for typical delays and not be too large to avoid needless extra waiting or travelling time for all passengers. In this consideration, the EETC function is a probabilistic weighted measurement of all cases in practice.

For a connection, the most desirable solution requires minimization of the local EETC; however, this is infeasible and impractical because of a variety of factors, such as resources, operation safety, and level of service, that affect the scheduled timetable. To overcome this problem, a robust transfer optimization model is developed by coordinating all of the local EETCs within a network as a whole such that practically applicable robust timetables can be obtained. The 
framework of the model is constructed depending on the topology of urban rail transit network, where the transfer arcs are directed and connected between the feeder trains nodes and the connection trains nodes, as illustrated via Figure 4 . The double buffer effect between running time supplement and transfer buffer time on each connection and the interactions between transfer buffer times on all connections would cause the interactions between running time supplements within network, and all the interaction effects determine the optimization and feasibility of the solution and play an essential and crucial role in coordinating all of the connections within the network.

4.1. Assumptions. Here, several assumptions are made throughout the paper for simplicity in the model formulation.

Assumption 1. The transfer walking time is assumed constant and fixed for all transferring passengers without allowing for the difference of passengers' age, sex, packages, etc., which mainly depends on the walking distances at transfer station and can be obtained by actual surveys.

Assumption 2. The path choices of passengers are known and fixed without considering the slight adjustment of the timetable plan. Although there may be alternative lines in the system to complete the travel, we do not consider them in this paper.

Assumption 3. The passenger flows are evenly distributed within a short time period. It is rather difficult to count the exact number of passengers getting on a train due to the randomness of the arrival time of passenger and the differences of headway between trains.

Assumption 4. The train person capacity is sufficient to accommodate all passengers, and passengers will always ride the first arriving train after reaching the connection platform. Although passengers may potentially encounter congestion delays at transfer stations in some specific cases, it is troublesome to formulate the passenger flows and timetable information, and thus we ignore the congestion effects.

\subsection{Nomenclature. Model parameters are defined as follows:}

$L$ : the set of lines in a network, $L=\{l \mid l=1,2, \cdots, n\}$, where $n$ is the total number of lines

$T_{l}$ : the set of trains on line $l, T_{l}=\left\{t \mid t=1,2, \cdots, k_{l}\right\}$, where $k_{l}$ is the total number of trains on line $l$

$I_{l}$ : the set of stations on line $l, I_{l}=\left\{i \mid i=1,2, \cdots, m_{l}\right\}$, where $m_{l}$ is the total number of stations on line $l$

$X$ : the set of connections in a network, $X=\{x \mid$ $\left.x=(l, t, i) \longrightarrow\left(l^{\prime}, t^{\prime}, i^{\prime}\right)\right\}$, where $(l, t, i) \longrightarrow\left(l^{\prime}, t^{\prime}, i^{\prime}\right)$ denotes train $t$ on line $l$ at station $i$, and train $t^{\prime}$ on line $l^{\prime}$ at station $i^{\prime}$ is a connection

$U$ : the set of connection origins, $U=\{u \mid u=$ $\left.(l, t, i),(l, t, i) \longrightarrow\left(l^{\prime}, t^{\prime}, i^{\prime}\right) \in X\right\}$

$h_{\min }^{l}, h_{\max }^{l}$ : the minimum and maximum headway on line $l$, respectively
$R_{i}^{l, t}:$ standard running time of train $t$ on line $l$ from station $i-1$ to station $i$

$W_{x}$ : transfer walking time on connection $x$

Variables of the proposed model are defined as follows:

$A_{i}^{l, t}$ : arrival time of train $t$ on line $l$ at station $i$

$D_{i}^{l, t}:$ departure time of train $t$ on line $l$ at station $i$

$D W_{i}^{l, t}:$ dwell time of train $t$ on line $l$ at station $i$

$S_{i}^{l, t}:$ running time supplement of train $t$ on line $l$ from station $i-1$ to station $i$

$B_{x}:$ transfer buffer time for connection $x$

\subsection{Constraints}

4.3.1. Resources Constraints. Generally, train operation follows a cyclic pattern of events: departure, running, arrival, and dwelling. Because of the continuity of these events, the departure time of a train at a station is the sum of the arrival time and dwell time at this station, as modeled via (11); meanwhile, the arrival time of a train at a station is the sum of the departure time at the first station, the total running time of the train, and dwell time at the previous station, as modeled via (12).

$$
\begin{aligned}
& D_{i}^{l, t}=A_{i}^{l, t}+D W_{i}^{l, t} \quad \forall l, t, i \\
& A_{i}^{l, t}=D_{0}^{l, t}+\sum_{j=1}^{i}\left(R_{j}^{l, t}+S_{j}^{l, t}\right)+\sum_{j=1}^{i-1} D W_{j}^{l, t} \quad \forall l, t, i
\end{aligned}
$$

Accordingly, modeling transfers requires consideration of the arrival time of the corresponding feeder train, transferring passenger walking time at transfer station, transferring passenger waiting time, and departure time of the connecting train. The transfer waiting time can be used as a buffer to prevent and counteract the effects of feeder train delays; therefore, the transfer buffer time is the difference between the departure time of the connecting train and the sum of the arrival time of the feeder train and transfer walking time, as modeled via

$$
\begin{aligned}
D_{i^{\prime}}^{l^{\prime}, t^{\prime}}-\left(A_{i}^{l, t}+W_{x}\right) & =B_{x} \\
& \forall x \in X, \quad x=(l, t, i) \longrightarrow\left(l^{\prime}, t^{\prime}, i^{\prime}\right)
\end{aligned}
$$

4.3.2. Operational Constraints. The constraint defined via (14) imposes a minimum headway $h_{\min }^{l}$ between departures of consecutive trains to guarantee operational safety, and a maximum headway $h_{\max }^{l}$ to maintain the standard level of service. Similarly, the constraint defined via (15) ensures that the dwell time is between a lower bound $d w_{i, \min }^{l}$ and an upper bound $d w_{i, \max }^{l}$. Besides, the departure times of the first train and the last train at first station on each line should be covered 


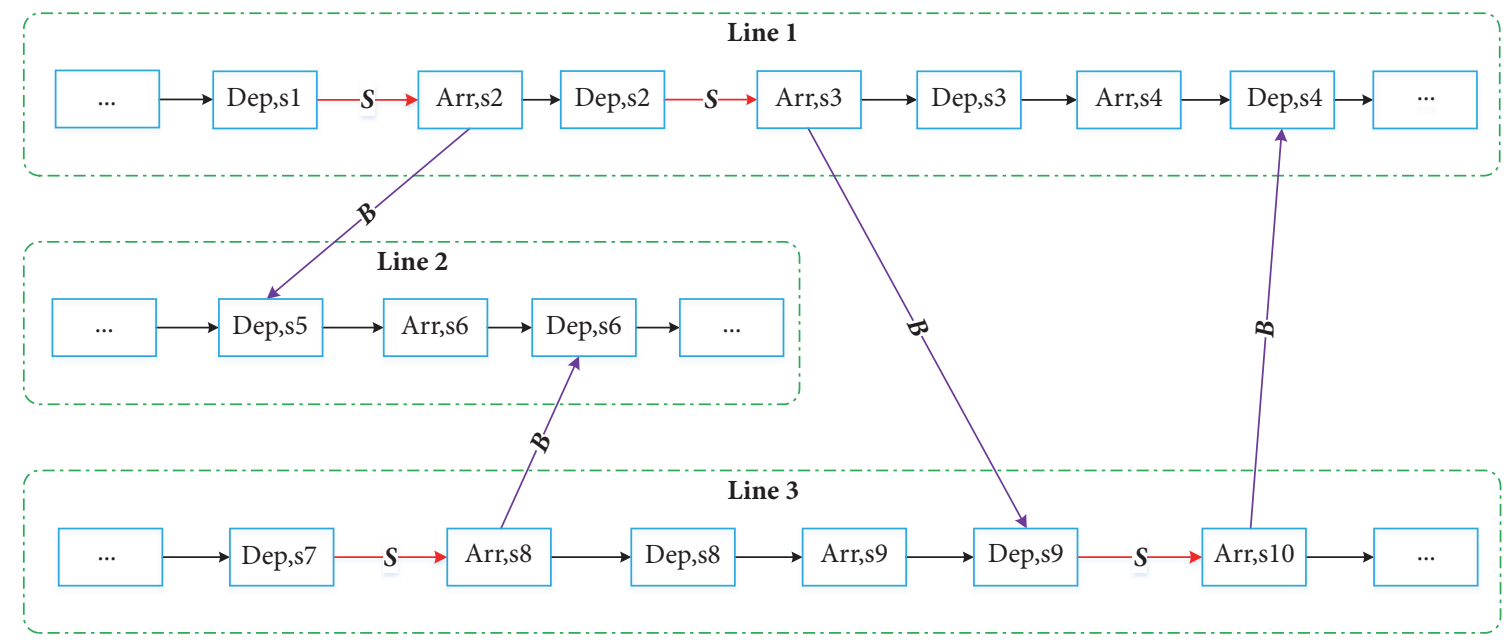

FIgURE 4: Framework of the robust transfer optimization model.

by the related planning horizon of each line, as demonstrated via (16).

$$
\begin{aligned}
h_{\min }^{l} & \leq D_{i}^{l, t}-D_{i}^{l, t-1} \leq h_{\max }^{l} \quad \forall l, t \geq 2, i \\
d w_{i, \min }^{l} & \leq D W_{i}^{l, t} \leq d w_{i, \max }^{l} \quad \forall l, t, i \\
D_{0}^{l, 1} & \geq D_{0, \min }^{l, t} ; \\
D_{0}^{l, k_{l}} & \leq D_{0, \max }^{l, t}
\end{aligned}
$$

$\forall l$

While the running time supplement is able to prevent delay propagation, an excessive supplement will reduce passenger satisfaction and the efficiency of rolling-stock usage. Thus, the acceptable range for the running time supplement is delimited below in (17); in addition, the constraint presented via (18) limits the total travel time of a train defined as the time travelling from the originating terminal station to the destination terminal station, which avoids inserting too much running time supplements in a line.

$$
\begin{aligned}
0 & \leq S_{i}^{l, t} \leq r_{i, \max }^{l} \quad \forall l, t, i \\
y_{\min }^{l} & \leq A_{m_{l}}^{l, t}-D_{0}^{l, t} \leq y_{\min }^{l} \quad \forall l, t
\end{aligned}
$$

4.4. Objective Function. The EETC function is weighted with the probabilities of all cases of delay to occur that incorporate the cases of successful and missed transfers with respect to transferring passengers and the cases of early-arriving and late-arriving with respect to nontransferring passengers, which should be a compromise between travel time extension and delay resistance risk. It is formulated based on the probability distributions of delay. The aim of robust transfer optimization is to decrease the EETC as much as possible by adjusting the running time supplement and transfer buffer time within the boundaries of the constraints described above. For this purpose, we implement the total EETC within a network that can be calculated by the summation of each connection's EETC (see (10)) as the objective to establish the robust transfer optimization model. Therefore, the objective function is given as follows:

$$
Q=\min \left(\sum_{x \in X} C_{x}\left(S_{i}^{l, t}, B_{x}\right)\right)
$$

\section{Approximate Linearization of Expected Extra Travel Cost}

As the objective function is nonlinear, solving the optimized model appears as a complex and difficult task. Thus, it is essential to determine an approach that is able to simplify the problem. Because linear systems typically exhibit features and properties that are considerably simpler, linearization of nonlinear functions is the most commonly used and effective method.

In this section, we first conduct a common and simple piecewise linearization of the local EETC and then discuss its feasibility and analyze the drawback that the approximated error of test points around the minimum local EETC is large and irregularly distributed. Then, to overcome the drawback, an improved piecewise linearization that, respectively, linearizes the EETC function for transferring and nontransferring passengers is proposed. The improved approach takes into account the characteristic of EETC and yields a higher goodness of fit. Finally, a further linearization of the objective via introduction of binary variables and auxiliary substitution variables is implemented to entirely convert the optimized model to a mixed integer linear programming model.

5.1. Piecewise Linearization. The common approximate linearization of a function is conducted via implementing the segmentation according to the general up or down trend of the function at first and then linearizing the function of each segment. A series of numerical tests shows that the local EETC can be approximated as a two-segment piecewise linear function, which yields higher goodness of fit and 
smaller errors than other types of approximation functions (e.g., continuous quadratic and cubic fitting function). As an example, we assume the following: $\lambda=0.03, h=600, P_{t r}=$ $300, P_{t h}=500, P_{d e}=P_{a r}=100$, with the function of the local EETC and its approximation shown in Figure 5. The red line located near the bottom of the left plot in Figure 5 is the threedimensional contour of the absolute value of the approximate error. The approximation function comprises two planes and is the approximate reflection of the general trend of the local EETC that is a continuous concave surface. Although clear advantages of piecewise linearization can be observed via the figure, the errors of test points around the minimum local EETC are significantly large.

The reason for this large error is that this approximation approach does not consider the double-buffer effect and thus regards it as the effect of proportional substitution for a certain proportion. More specifically, the effects of a 1 min. running time supplement on local EETC are equivalent to that of a 1.2 min. transfer buffer time. To correct this problem and achieve a higher goodness of fit, an improved linearization technique that takes into account all factors affecting the EETC is proposed.

5.2. Improvement of Piecewise Linearization. As previously mentioned, while both the transfer buffer time and running time supplement affect the EETC of transferring passengers, the EETC of nontransferring passengers is only affected by the running time supplement. More importantly, the effects of each type of buffer time are equivalent for transferring passengers; this suggests that an alternative variable $S+B$ can be used to represent the double-buffer effect. Thus, the local EETC can be divided into two categories: (1) the EETC of transferring passengers and (2) the EETC of nontransferring passengers. These two categories can then be approximately linearized independently of each other, as expressed via (20) and (21) for transferring and nontransferring passengers, respectively.

$$
\begin{aligned}
& C_{T r} \approx \tilde{g}(S, B) \\
& = \begin{cases}\tilde{g}^{1}(S, B)=b_{0}^{1}+b_{1}^{1} \times(S+B), & \tilde{g}^{2}(S, B)<\tilde{g}^{1}(S, B) \\
\tilde{g}^{2}(S, B)=b_{0}^{2}+b_{1}^{2} \times(S+B), & \tilde{g}^{2}(S, B) \geq \tilde{g}^{1}(S, B)\end{cases} \\
& C_{N \text { No- } T r} \approx \tilde{f}(S) \\
& = \begin{cases}\tilde{f}^{1}(S)=a_{0}^{1}+a_{1}^{1} \times S, & \tilde{f}^{2}(S)<\tilde{f}^{1}(S) \\
\tilde{f}^{2}(S)=a_{0}^{2}+a_{1}^{2} \times S, & \tilde{f}^{2}(S) \geq \tilde{f}^{1}(S)\end{cases}
\end{aligned}
$$

Figure 6 illustrates the contour plots of the EETC and the improved EETC approximation, which is calculated by performing separate linearization approximations for each of the two categories of EETC; the assumptions applied in the previous example in Section 5.1 were applied $(\lambda=0.03, h=$ $\left.600, P_{t r}=300, P_{t h}=500, P_{d e}=P_{a r}=100\right)$. The red line located near the bottom of the left plot in Figure 6 is the three-dimensional contour of the absolute value of the approximate error. From the figure, it can be determined that the independent linearization technique yields a better goodness of fit, which is evidenced by decreased error about the minimum local EETC. The contour of the absolute value of the error is observed as constrained, regular, and ordered, thereby suggesting that the improved piecewise linearization technique can provide a more accurate description of the up or down trends of the local EETC. These results demonstrate that the linearization technique presented in this paper effectively represents and explains the characteristics of the EETC function.

In summary, the ordinary approximation approach simplifies the relationship between the effects of the running time supplement and transfer buffer time, which causes the large and irregularly distributed errors of test points around the minimum local EETC. However, the improved approach takes into account both the difference between the effects of buffer time on transferring and nontransferring passengers and the fact that the running time supplement and transfer buffer time yield equivalent effects on transferring passengers. In this way, even though the errors do exist, the trend, up or down, of the local EETC can be captured with reasonable accuracy.

5.3. Objective Conversion. Although the linearization approximation technique proposed here yields improvements, the EETC of nontransferring passengers still pose a problem, as it may be included in computations more than once, as demonstrated in Figure 7. More specifically, Figure 7 illustrates Train A as having two connections at the transfer station: Connections 1 and 2. In this case, the EETCs of nontransferring passengers are identical for the two connections. Considering this, if the total EETC is a simple sum of local EETCs, although the EETCs are identical and therefore need to only be considered once as a single EETC, they will receive duplicate consideration in computation. This concept is also applicable in the cases of nontransferring passengers on Trains B, C, and D. When three or more lines intersect, it could be coped with in the same way and the EETC of nontransferring passengers only needs to be counted once.

Therefore, to avoid duplicated consideration, we define a set of transfer connection points of origin $U, U=\{u$ | $\left.u=(l, t, i),(l, t, i) \longrightarrow\left(l^{\prime}, t^{\prime}, i^{\prime}\right) \in X\right\}$, to calculate the EETC of transferring and nontransferring passengers separately. In this consideration, the objective function (19) can then be expressed by formula (22).

$$
\begin{aligned}
& \text { Objective } \\
& \quad=\min \left(\sum_{u \in U} C_{N o n-T r, u}\left(S_{i}^{l, t}\right)+\sum_{x \in X} C_{T r, x}\left(S_{i}^{l, t}, B_{x}\right)\right) \\
& \approx \min \left(\sum_{u \in U} \tilde{f}_{u}\left(S_{i}^{l, t}\right)+\sum_{x \in X} \tilde{g}_{x}\left(S_{i}^{l, t}, B_{x}\right)\right)
\end{aligned}
$$

Although the function $\tilde{f}_{\mathcal{u}}\left(S_{i}^{l, t}\right)$ has been linearized, because $\tilde{g}_{x}\left(S_{i}^{l, t}, B_{x}\right)$ remains to be a nonlinear function, the optimized model is still nonlinear. Considering this, several binary variables and auxiliary substitution variables 

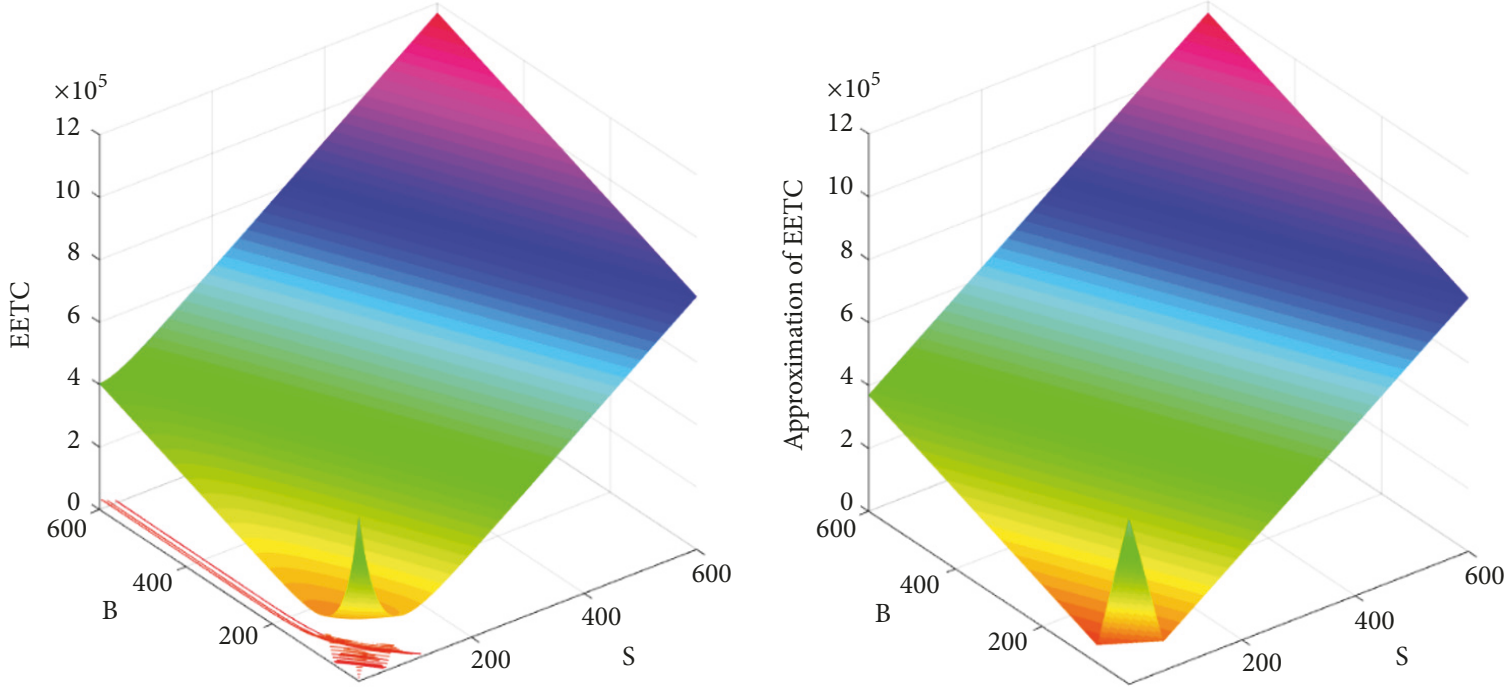

Figure 5: Contour plots of the local and approximate EETC.
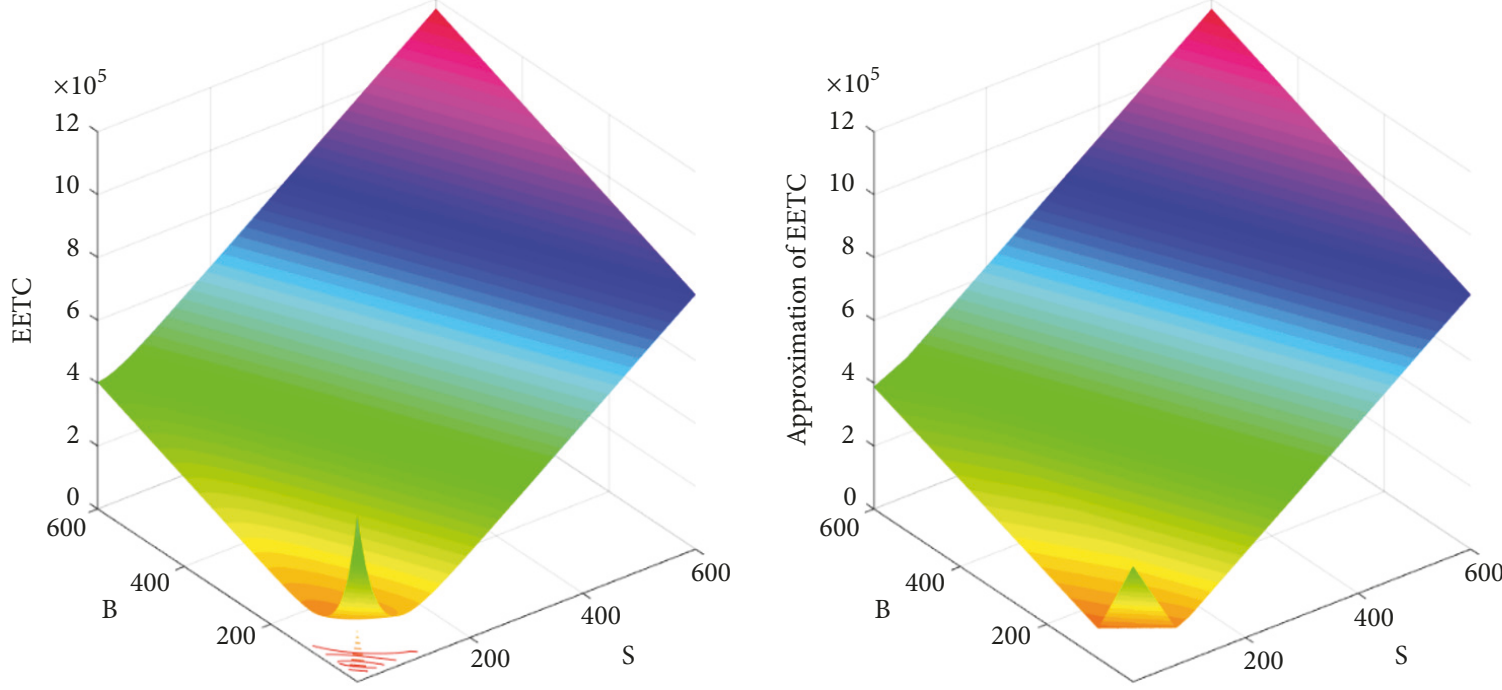

FIgURE 6: Contour plots of the local and improved approximate EETC.

are introduced to fully linearize the objective function (22), which can facilitate the optimized model solving.

5.3.1. Approximation Function $\tilde{f}_{u}\left(S_{i}^{l, t}\right)$. The constraint presented as (23) activates the binary variable $\delta_{u}$. If $\widetilde{f}_{u}^{2}\left(S_{i}^{l, t}\right)<$ $\tilde{f}_{u}^{1}\left(S_{i}^{l, t}\right), \delta_{u}$ is equal to 0 ; otherwise it is equal to 1 . Then, the approximation function $\tilde{f}_{u}\left(S_{i}^{l, t}\right)$ for nontransferring passengers can be expressed via a piecewise function, as presented in (24), where $M$ is a large positive integer.

$$
\begin{aligned}
M\left(\delta_{u}-1\right) & \leq \widetilde{f}_{u}^{2}\left(S_{i}^{l, t}\right)-\tilde{f}_{u}^{1}\left(S_{i}^{l, t}\right) \leq M \delta_{u} \\
\tilde{f}_{u}\left(S_{i}^{l, t}\right) & =\left(1-\delta_{u}\right) \times \widetilde{f}_{u}^{1}\left(S_{i}^{l, t}\right)+\delta_{u} \times \widetilde{f}_{u}^{2}\left(S_{i}^{l, t}\right)
\end{aligned}
$$

Because (23) is a nonlinear function with a cross product of decision variables, we introduce auxiliary substitution variables $A S_{u}^{1}, A S_{u}^{2}$ to construct (25) and (26) for subsequent substitution into (23). In addition, the approximation function $\tilde{f}_{u}\left(S_{i}^{l, t}\right)$ can be substituted with $A S_{u}^{1}+A S_{u}^{2}$.

$$
\begin{aligned}
\widetilde{f}_{u}^{1}\left(S_{i}^{l, t}\right) & \leq A S_{u}^{1}+M \delta_{u}, \\
\widetilde{f}_{u}^{2}\left(S_{i}^{l, t}\right) & \leq A S_{u}^{2}+M\left(1-\delta_{u}\right) \\
0 & \leq A S_{u}^{1}+M\left(1-\delta_{u}\right), \\
0 & \leq A S_{u}^{2}+M \delta_{u}
\end{aligned}
$$

5.3.2. Approximation Function $\widetilde{g}_{x}\left(S_{i}^{l, t}, B_{x}\right)$. Although $\widetilde{g}_{x}\left(S_{i}^{l, t}\right.$, $B_{x}$ ) is a function with two variables, $S_{i}^{l, t}+B_{x}$ can be taken as a new and alternative variable because they each yield identical effects. Similarly, we can formulate (27)-(29) by introducing 


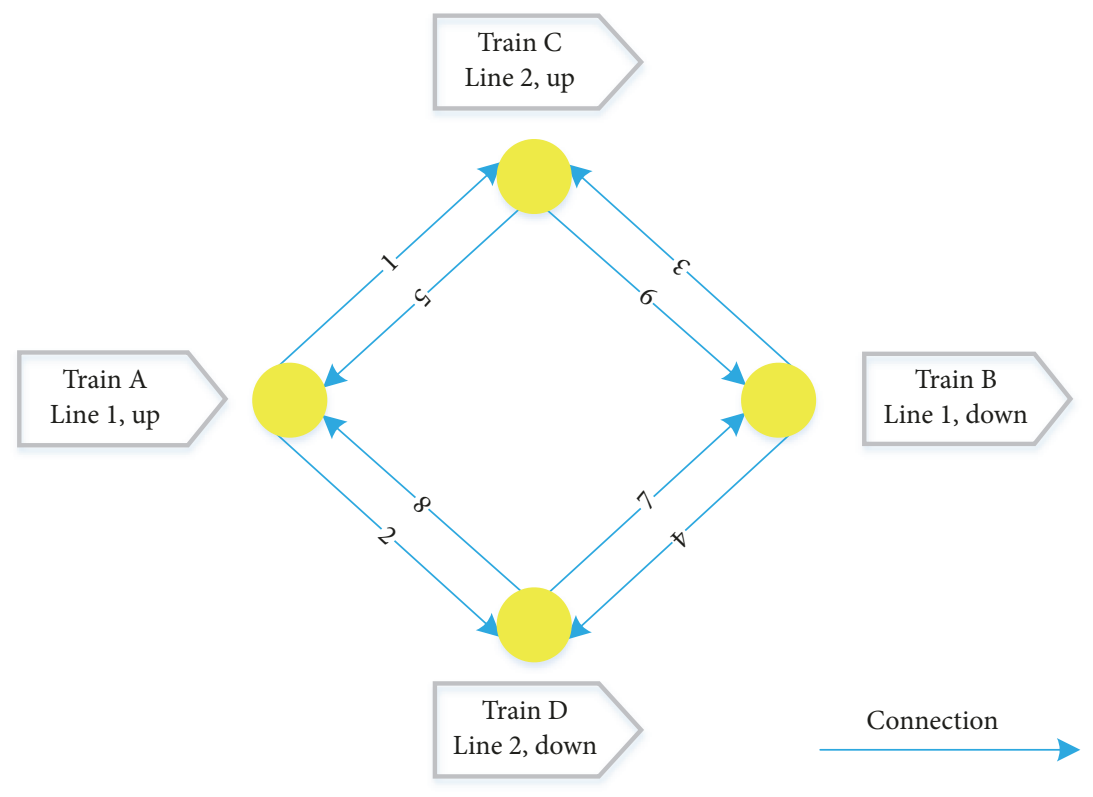

FIGURE 7: Duplicate consideration of nontransferring passenger EETC.

binary variables $\varphi_{x}$ and auxiliary substitution variables $A S_{x}^{3}$, $A S_{x}^{4}$. The approximation function $\tilde{g}_{x}\left(S_{i}^{l, t}, B_{x}\right)$ can then be substituted with $A S_{x}^{3}+A S_{x}^{4}$.

$$
\begin{aligned}
M\left(\varphi_{x}-1\right) & \leq \widetilde{g}_{x}^{2}\left(S_{i}^{l, t}, B_{x}\right)-\tilde{g}_{x}^{2}\left(S_{i}^{l, t}, B_{x}\right) \leq M \varphi_{x} \\
\tilde{g}_{x}^{1}\left(S_{i}^{l, t}, B_{x}\right) & \leq A S_{x}^{3}+M \varphi_{x}, \\
\tilde{g}_{x}^{2}\left(S_{i}^{l, t}, B_{x}\right) & \leq A S_{x}^{4}+M\left(1-\varphi_{x}\right) \\
0 & \leq A S_{x}^{3}+M\left(1-\varphi_{x}\right), \\
0 & \leq A S_{x}^{4}+M \varphi_{x}
\end{aligned}
$$

5.3.3. Approximation of Objective Function. Subsequently, the objective function can be expressed as (30), which then enables conversion of the proposed model to a mixedinteger linear programming model that can be solved via any linear programming software, such as Cplex or Gurobi. The variables of the optimized model are separated into three different types: (1) timetable variables $A_{i}^{l, t}, D_{i}^{l, t}, D W_{i}^{l, t}, S_{i}^{l, t}$, and $B_{u}$; (2) binary variables $\delta_{u}$ and $\varphi_{x}$; and (3) auxiliary substitution variables $A S_{u}^{1}, A S_{u}^{2}, A S_{x}^{3}$, and $A S_{x}^{4}$.

\section{Objective}

$$
=\min \left(\sum_{u \in U}\left(A S_{u}^{1}+A S_{u}^{2}\right)+\sum_{x \in X}\left(A S_{x}^{3}+A S_{x}^{4}\right)\right)
$$

\section{Experimental Simulation}

6.1. Description. In this section, a test network comprising four bidirectional subway lines ( 8 unidirectional subway lines) and 5 transfer stations is created and used to validate the performance of our proposed model and the improved linearization approach (Figure 8). In addition, the primary train delays are assumed to yield a negative exponential distribution, and the mean delay times are approximately one-fifth of the standard running times. The mean headways for Lines 1, 2, 7, and 8 are $15 \mathrm{~min}$., while those for Lines 3, 4, 5 , and 6 are $8 \mathrm{~min}$.

6.2. Comparison Schemes. In order to demonstrate the advantages of the proposed method, of which the resulting optimized timetable is designated as RobT, we design two other types of buffer schemes for the purpose of comparison: (1) the proportional buffer scheme (k\%-ProT) and (2) the optimal buffer scheme that consider only the effects of the running time supplement (RobT-S).

(1) k\%-ProT: for actual operation, the commonly implemented method to improve timetable robustness and absorb delay is to define the running time supplement as a certain proportion (e.g., $4 \%-10 \%$ ) of the standard running time. For the $\mathrm{k} \%$-ProT scheme, the running time supplement is $k$ percent of its standard running time. This is a simple method.

(2) RobT-S: this RobT-S scheme is based on the commonly applied concept of adjusting only the running time supplement and is subsequently solved by minimizing the EETC, which is determined by assuming the transfer buffer time is zero in the objective function.

6.3. Optimized Results. The optimal scheme and comparison schemes are all generated via $\mathrm{C \#}$, and computational results are presented by using the integer linear solver Cplex 12.5 on a desktop computer (Intel Pentium CPU G3240 $3.1 \mathrm{GHz}$, 


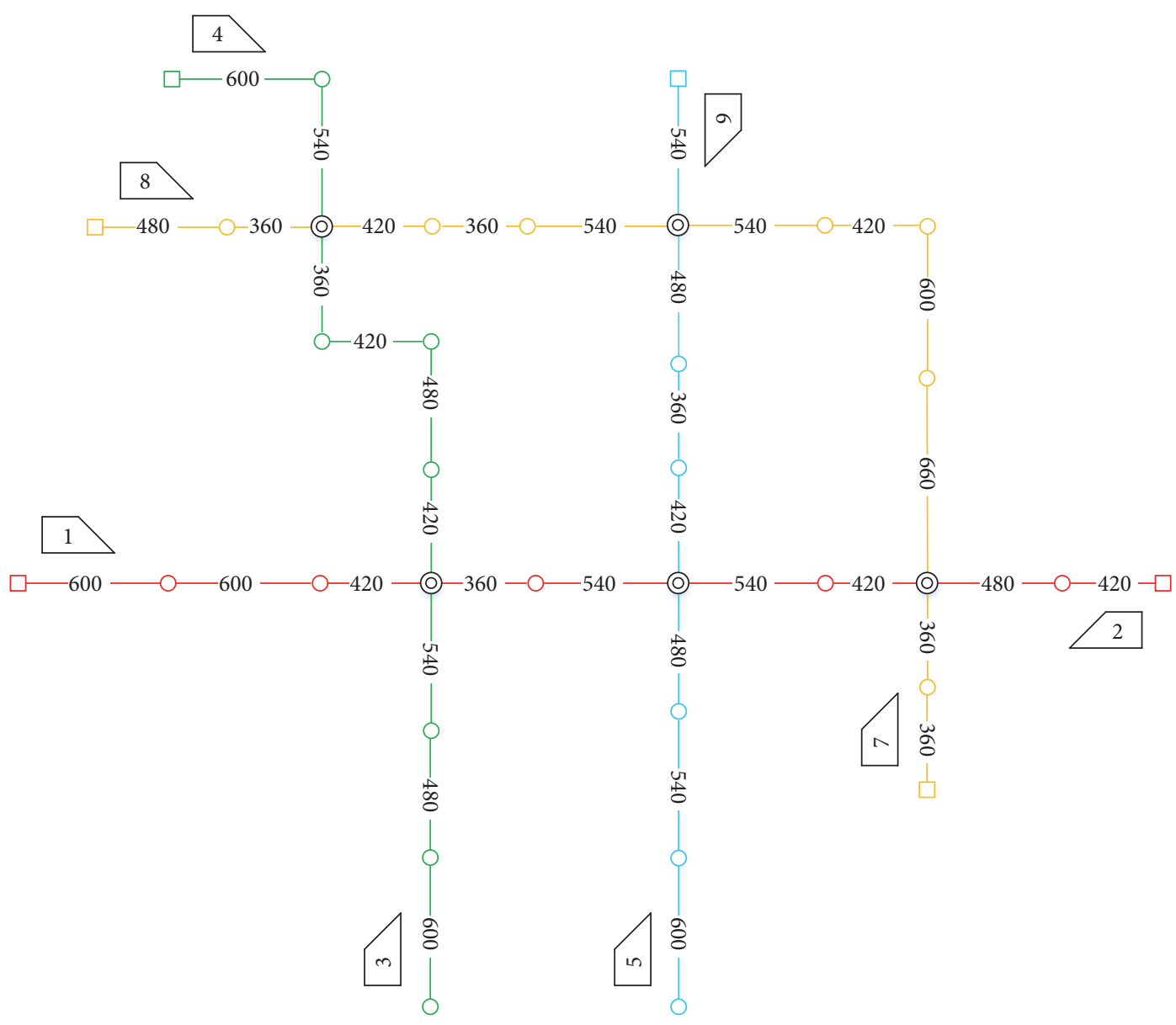

FIGURE 8: Topology of the test network.

4 GB RAM). The EETC and App_EETC are the actual and approximate EETC of all passengers within the network, respectively. Besides, 1000 simulation scenarios are randomly generated based on the delay probability distributions. The proportion of missed transfer passengers (PMTP) for each buffer scheme is computed via discrete-event simulation and can be used as a measure to evaluate the timetable. The optimized results are shown in Table 2.

Among these buffer schemes, the RobT scheme is the most efficient, as it yields the minimum EETC and a small and acceptable PMTP. By comparison, the RobT-S scheme exhibits inferior performance with respect to both EETC and PMTP; this indicates that the efficiency and robustness of the timetable can be improved by appropriately allocating the running time supplement and transfer buffer time. On the other hand, the RobT scheme yields a fine approximate error $(3.601-3.432) \div 3.432=4.92 \%$, while the RobT-S scheme does badly in approximation with an approximate error (5.364$4.458) \div 4.458=20.32 \%$. This noticeable error stems from two reasons: ignorance of the effect of transfer buffer time that generates fundamental deviation from actual EETC and further limits in approximation method that produces approximate error. On the whole, even though the approximate error of RobT scheme is not small enough, it is still enough to provide superior description of the trends of optimization objective.

In addition, the robustness of the $\mathrm{k} \%$-ProT schemes increases with increased proportion $\mathrm{k} \%$. When $\mathrm{k}=0 \%$, the PMTP is substantially high and the EETC is also relatively high. However, although the PMTP is reduced to the smallest value for $\mathrm{k}=20 \%$, the EETC still remains high; this is indicative of the fact that a tiny and an excess of buffer time are both uneconomical.

In summary, because the $\mathrm{k} \%$-ProT schemes disregard passenger characteristics and the effects of buffer time, it is unable to appropriately balance efficiency and robustness. Moreover, the RobT-S scheme does not consider the doublebuffer effect, thereby yielding a less practical EETC. Furthermore, our proposed model can significantly improve the robustness of the timetable by concurrently adjusting the running time supplement and transfer buffer time, while yielding only a small PMTP.

6.3.1. EETC of Nontransferring and Transferring Passengers. Figure 9 shows the EETC histogram for transferring and nontransferring passengers. As can be seen, for $\mathrm{k} \%$-ProT schemes, with respect to increasing proportion $k \%$, the EETC of nontransferring passengers exhibits a trend of 
TABle 2: Total EETC, App_EETC, and PMTP for different buffer schemes.

\begin{tabular}{|c|c|c|c|c|c|c|c|}
\hline \multirow{2}{*}{ Scheme } & \multirow{2}{*}{ RobT } & \multirow{2}{*}{ RobT-S } & \multicolumn{5}{|c|}{ k\%-ProT } \\
\hline & & & $\mathrm{k}=0$ & $\mathrm{k}=5$ & $\mathrm{k}=10$ & $\mathrm{k}=15$ & $\mathrm{k}=20$ \\
\hline $\operatorname{EETC~}\left(* 10^{7} \mathrm{~s}\right)$ & 3.432 & 4.458 & 6.287 & 4.468 & 4.192 & 4.569 & 5.131 \\
\hline App_EETC $\left(* 10^{7} \mathrm{~s}\right)$ & 3.601 & 5.364 & - - & -- & -- & -- & -- \\
\hline PMTP & $2.311 \%$ & $3.375 \%$ & $17.423 \%$ & $12.572 \%$ & $5.875 \%$ & $5.004 \%$ & $0.820 \%$ \\
\hline
\end{tabular}

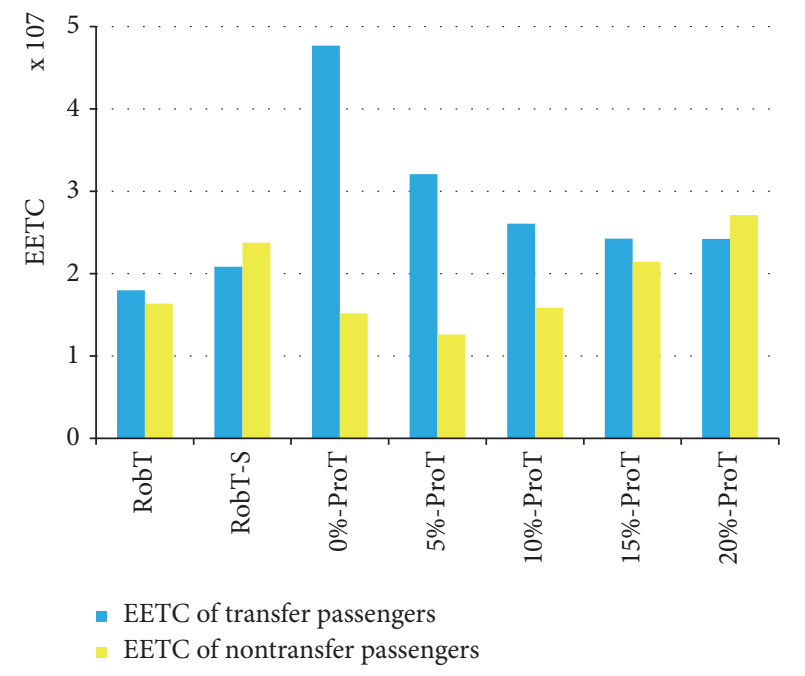

FIGURE 9: EETC histogram for transferring and nontransferring passengers.

initial decrease and subsequent increase. This trend occurs because a moderate running time supplement can improve the timetable flexibility that allows more freedom at the operational level to arrive or depart flexibly and compensate for typical delays. As there is a higher VOT under the delay circumstances, the timetable flexibility can bring about a reduction in the EETC of nontransferring passengers. However, when the proportional value of the standard running time exceeds a certain value, it will become excessively and needless redundant and reduce the efficiency of the timetable, thereby leading to an increase in the EETC of nontransferring passengers.

In contrast to that of nontransferring passengers, the EETC of transferring passengers continuously decreases with increased proportion $k \%$. This is because the timetable for the $\mathrm{k} \%$-ProT scheme is a synchronization timetable aiming to minimize the total passenger transfer waiting time and the possibilities of delay are not considered. Hence, the transfer buffer times tend to be as small as possible for the sake of efficiency (for example, zero if there are no conflicts). Furthermore, because of the long headway on the connecting line, the running time supplement can be used as a buffer to avoid missing transfers, as well as to reduce the EETC of transferring passengers.

In addition, as compared to the RobT-S scheme, although the increased EETC of transferring passengers with respect to the RobT scheme is not significant, the increase in EETC of nontransferring passengers is relatively large. Thus, from the perspective of passengers within a network, because disregarding the double-buffer effect is demonstrated to increase the EETC of nontransferring passengers, the RobT$\mathrm{S}$ scheme is shown to be an inefficient scheme. As a result, it is of great significance to consider the double-buffer effect by concurrently adjusting the running time supplement and transfer buffer time to generate a robust, yet efficient, timetable.

6.3.2. Missed-Transfer Passengers. Figure 10 illustrates the distribution of the missed-transfer passengers for three different buffer schemes under 1000 random simulation scenarios. In general, a small mean and fluctuation of the missed-transfer passengers are indicative of a fine recoverable robustness such that the scheduled timetables are easier to recover by taking a set of recoverable actions. The concept of recoverable robustness combines the optimization of the scheduled timetable and the strategy of recovery. As can be seen, when there is no running time supplement, the $0 \%$ ProT scheme exhibits poor performance with respect to both the mean and fluctuation of the missed-transfer passengers. Conversely, the RobT scheme results in a small number of passengers missing their transfer, thereby also implying small fluctuations in this amount, which is comparable with the 20\%-ProT scheme. This implies that the timetable generated via the proposed method is more robust and stable in the event of a delay.

\section{Conclusions}

To date, the primary objective when solving train timetabling optimization problems has been to improve the robustness by only adjusting the running time supplement. However, in this paper, we not only consider both the running time supplement and the transfer buffer time and concurrently adjust them to increase efficiency. The main contributions of this paper can be summarized as follows:

(1) This paper demonstrates analysis of the effects of the two types of buffer times on train delays and formulates the nonlinear function for expected extra travel cost (EETC) that includes the running time supplement and transfer buffer time.

(2) To coordinate all the connections within a network as a whole, a robust transfer optimization model is constructed by minimizing the total EETC. The resulting objective function that is a compromise between travel time extension and delay resistance risk can be used to appropriately balance efficiency and robustness in practice. 


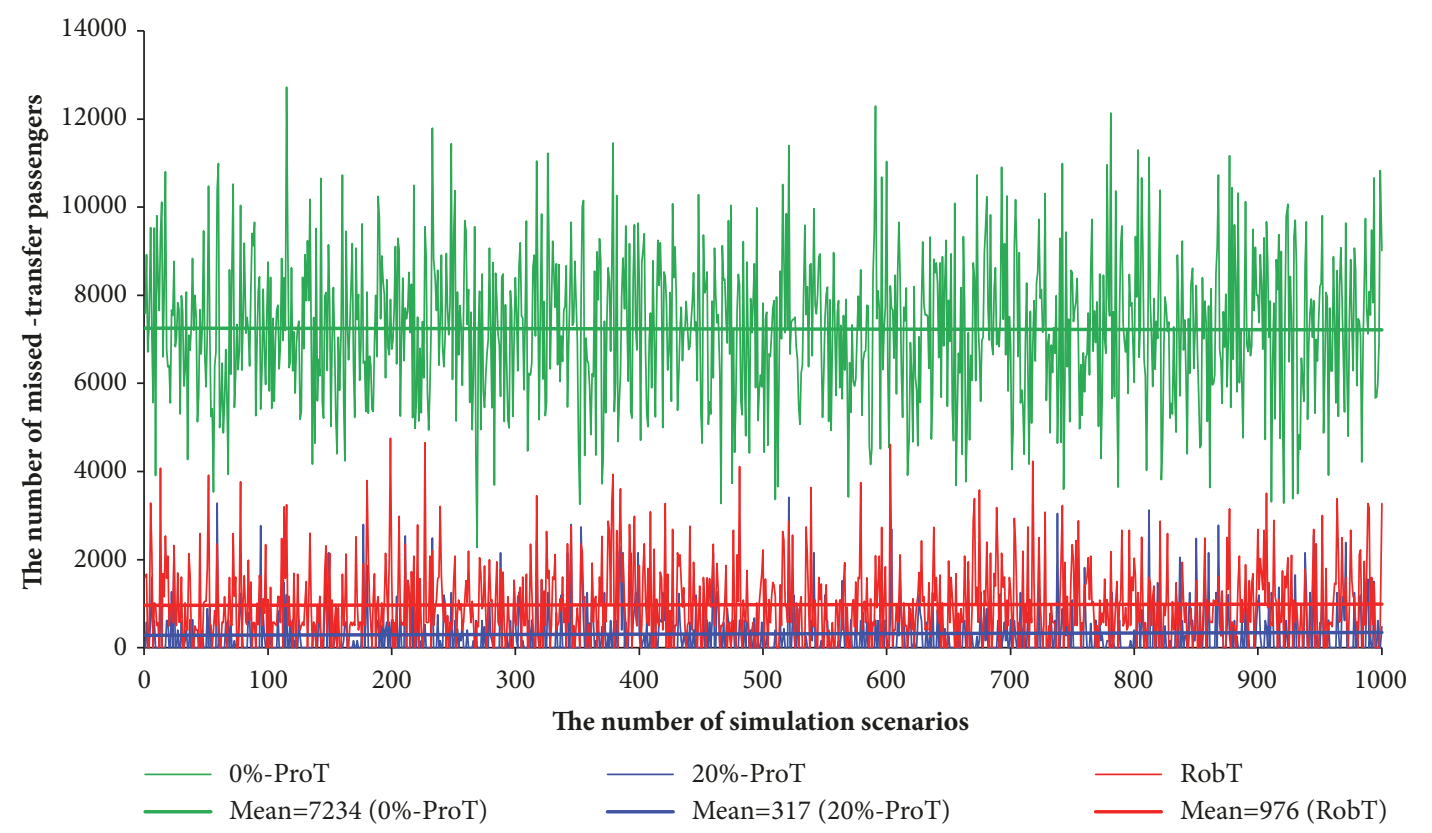

FIgURE 10: Distribution of the missed-transfer passengers.

(3) To simplify and enhance the efficiency of the model, we propose an improved approximate linearization approach that is based on characteristics of the EETC; we then introduce several binary variables and auxiliary substitution variables to fully linearize the EETC, enabling the proposed model to be solved via linear programming software.

Furthermore, experimental simulations are conducted to demonstrate that our proposed method can generate robust yet efficient solutions. It is shown that the proportional buffer schemes do not consider the characteristics associated with each type of passenger and that the buffer scheme only considers the effects of running time supplement; these oversights each yield impractical expectations of overall extra travel cost. Because the performances of both are shown to be inferior to our optimized scheme, we have proven that it is of vital importance to optimize timetable robustness by considering both the running time supplement and transfer buffer time.

Some extension is suggested for future research. First, the approximate linearization approach can be improved by introducing a multisegment, more than two segments, piecewise linear function to obtain a higher goodness of fit. However, the computational times may be affected. Second, although our proposed approach is based on fixed transfer pairs, the main idea can be implemented to integrate connection precedence coordination and transfer robustness optimization. Further research might also address applicable and efficient ways to generate the integrated solutions.

\section{Data Availability}

The data used to support the findings of this study are included within the article.

\section{Conflicts of Interest}

The authors declare that they have no conflicts of interest.

\section{Acknowledgments}

This work was supported by the Fundamental Research Funds for the Central Universities (grant number 2018YJS074), the National Natural Science Foundation of China (grant number 51478036), and the Talent Fund Project of Beijing Jiaotong University (grant number 2017RC036).

\section{References}

[1] M. A. Salido, F. Barber, and L. Ingolotti, "Robustness for a single railway line: Analytical and simulation methods," Expert Systems with Applications, vol. 39, no. 18, pp. 13305-13327, 2012.

[2] A. Landex, Methods to Estimate Railway Capacity and Passenger Delays, Technical University of Denmark, 2008.

[3] T. Dollevoet, F. Corman, A. D’Ariano, and D. Huisman, "An iterative optimization framework for delay management and train scheduling," Flexible Services \& Manufacturing Journal, vol. 26, no. 4, pp. 490-515, 2014.

[4] F. Corman, A. D’Ariano, and I. A. Hansen, "Evaluating disturbance robustness of railway schedules," Journal of Intelligent Transportation Systems: Technology, Planning, and Operations, vol. 18, no. 1, pp. 106-120, 2014.

[5] J. Parbo, O. A. Nielsen, and C. G. Prato, "Passenger Perspectives in Railway Timetabling: A Literature Review," Transport Reviews, vol. 36, no. 4, pp. 500-526, 2016.

[6] V. Cacchiani and P. Toth, "Nominal and robust train timetabling problems," European Journal of Operational Research, vol. 219, no. 3, pp. 727-737, 2012. 
[7] L. Kroon, G. Maróti, M. R. Helmrich, M. Vromans, and R. Dekker, "Stochastic improvement of cyclic railway timetables," Transportation Research Part B: Methodological, vol. 42, no. 6, pp. 553-570, 2008.

[8] P. Jovanović, P. Kecman, N. Bojović, and D. Mandić, "Optimal allocation of buffer times to increase train schedule robustness," European Journal of Operational Research, vol. 256, no. 1, pp. 4454, 2017.

[9] M. Fischetti and M. Monaci, "Light robustness," Lecture Notes in Computer Science (including subseries Lecture Notes in Artificial Intelligence and Lecture Notes in Bioinformatics): Preface, vol. 5868, pp. 61-84, 2009.

[10] A. D'Ariano, D. Pacciarelli, and M. Pranzo, "Assessment of flexible timetables in real-time traffic management of a railway bottleneck," Transportation Research Part C: Emerging Technologies, vol. 16, no. 2, pp. 232-245, 2008.

[11] C. Liebchen, M. Lübbecke, R. Möhring, and S. Stiller, "The Concept of Recoverable Robustness, Linear Programming Recovery, and Railway Applications," in Robust and Online Large-Scale Optimization, vol. 5868 of Lecture Notes in Computer Science, pp. 1-27, Springer Berlin Heidelberg, Berlin, Heidelberg, 2009.

[12] G. D’Angelo, G. Di Stefano, A. Navarra, and C. M. Pinotti, "Recoverable robust timetables: an algorithmic approach on trees," Institute of Electrical and Electronics Engineers. Transactions on Computers, vol. 60, no. 3, pp. 433-446, 2011.

[13] M. Goerigk and A. Schöbel, "Recovery-to-optimality: A new two-stage approach to robustness with an application to aperiodic timetabling," Computers \& Operations Research, vol. 52, pp. 1-15, 2014.

[14] M. Samà, A. D’Ariano, F. Corman, and ETAL, "A variable neighborhood search for fast train scheduling and routing during disturbed railway traffic situations," Computers \& Operations Research, vol. 97, no. 1, pp. 142-156, 2016.

[15] E. V. Andersson, A. Peterson, and J. Törnquist Krasemann, "Reduced railway traffic delays using a MILP approach to increase Robustness in Critical Points," Journal of Rail Transport Planning and Management, vol. 5, no. 3, pp. 110-127, 2015.

[16] T. Schlechte and R. Borndörfer, "Balancing Efficiency and Robustness - A Bi-criteria Optimization Approach to Railway Track Allocation," in Multiple Criteria Decision Making for Sustainable Energy and Transportation Systems, vol. 634 of Lecture Notes in Economics and Mathematical Systems, pp. 105116, Springer Berlin Heidelberg, Berlin, Heidelberg, 2010.

[17] V. Cacchiani, A. Caprara, and M. Fischetti, "A lagrangian heuristic for robustness, with an application to train timetabling," Transportation Science, vol. 46, no. 1, pp. 124-133, 2012.

[18] Y. Lee, L.-S. Lu, M.-L. Wu, and D.-Y. Lin, "Balance of efficiency and robustness in passenger railway timetables," Transportation Research Part B: Methodological, vol. 97, pp. 142-156, 2017.

[19] A. O. Nielsen, A. Landex, and R. D. Frederiksen, "Passenger delay models for rail networks," in Schedule-Based Modeling of Transportation Networks: Theory and applications, vol. 46, pp. 46-27, 2008.

[20] S. Kanai, K. Shiina, S. Harada, and N. Tomii, "An optimal delay management algorithm from passengers' viewpoints considering the whole railway network," Journal of Rail Transport Planning and Management, vol. 1, no. 1, pp. 25-37, 2011.
[21] F. Corman, A. D’Ariano, D. Pacciarelli, and M. Pranzo, "Biobjective conflict detection and resolution in railway traffic management," Transportation Research Part C: Emerging Technologies, vol. 20, no. 1, pp. 79-94, 2012.

[22] M. M. Nesheli and A. A. Ceder, "A robust, tactic-based, realtime framework for public-transport transfer synchronization," Transportation Research Part C: Emerging Technologies, vol. 60, pp. 105-123, 2015.

[23] C. Liebchen, M. Schachtebeck, A. Schöbel, S. Stiller, and A. Prigge, "Computing delay resistant railway timetables," Computers \& Operations Research, vol. 37, no. 5, pp. 857-868, 2010.

[24] C. Liebchen and S. Stiller, "Delay resistant timetabling," Public Transport, vol. 1, no. 1, pp. 55-72, 2009.

[25] A. Schöbel and A. Kratz, "A Bicriteria Approach for Robust Timetabling," in Robust and Online Large-Scale Optimization, vol. 5868 of Lecture Notes in Computer Science, pp. 119-144, Springer Berlin Heidelberg, Berlin, Heidelberg, 2009.

[26] P. Vansteenwegen and D. Van Oudheusden, "Developing railway timetables which guarantee a better service," European Journal of Operational Research, vol. 173, no. 1, pp. 337-350, 2006.

[27] P. Vansteenwegen and D. Van Oudheusden, "Decreasing the passenger waiting time for an intercity rail network," Transportation Research Part B: Methodological, vol. 41, no. 4, pp. 478492, 2007.

[28] T. Dewilde, "Defining robustness of a railway timetable," in Proceedings of International Seminar on Railway Operations Modelling Analysis, pp. 108-109, Rome, Italy, Railrome, 2011.

[29] P. Sels, T. Dewilde, D. Cattrysse, and P. Vansteenwegen, "Reducing the passenger travel time in practice by the automated construction of a robust railway timetable," Transportation Research Part B: Methodological, vol. 84, pp. 124-156, 2016.

[30] R. M. P. Goverde, "Optimal Scheduling of Connections in Railway Systems," in Proceedings of the World Conference on Transport Research, p. a, Antwerp, Belgium, 1998.

[31] P. Weston, J. Preston, and R. Takagi, "Minimising train delays in a mixed traffic railway network with consideration of passenger delay," in Proceedings of the World Congress on Railway Research, Montreal, Canada, 2006.

[32] R. M. P. Goverde, "Max-plus algebra approach to railway timetable design," in Proceedings of the 1998 6th International Conference on Computer Aided Design, Manufacture and Operation in the Railway and Other Advanced Mass Transit Systems, pp. 339-350, September 1998.

[33] J. Yuan, M. P. R. Goverde, and I. A. Hansen, Propagation of Train Delays in stations, Publication of Wit Press, 2002.

[34] J. Yuan, R. M. Goverde, and I. A. Hansen, "Evaluating stochastic train process time distribution models on the basis of empirical detection data," in Proceedings of the COMPRAIL 2006, pp. 631640, Prague, Czech Republic, July 2006.

[35] N. Marković, S. Milinković, K. S. Tikhonov, and P. Schonfeld, "Analyzing passenger train arrival delays with support vector regression," Transportation Research Part C: Emerging Technologies, vol. 56, pp. 251-262, 2015.

[36] H. Mohring, J. Schroeter, and P. Wiboonchutikula, "The values of waiting time, travel time, and a seat on the bus," The RAND Journal of Economics, vol. 18, no. 1, pp. 40-56, 1987. 
[37] H. Gunn F, J. Tuinenga G, Y. Cheung et al., "Value of Dutch travel time savings in 1997," in Proceedings of the World Transport Research: Selected Proceedings of the 8th World Conference on Transport Research, Antwerp, Belgium, 1999.

[38] M. Wardman, J. Hine, and S. Stradling, Interchange and Travel Choice: Volume 1. Transport Research, Stradling S. Interchange and Travel Choice, Volume 1. Transport Research, 2001.

[39] M. Wardman, "Public transport values of time," Transport Policy, vol. 11, no. 4, pp. 363-377, 2004. 


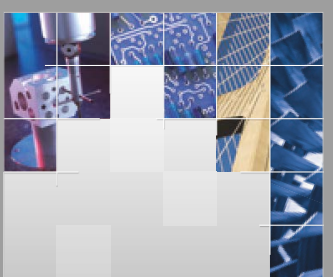

\section{Enfincering}
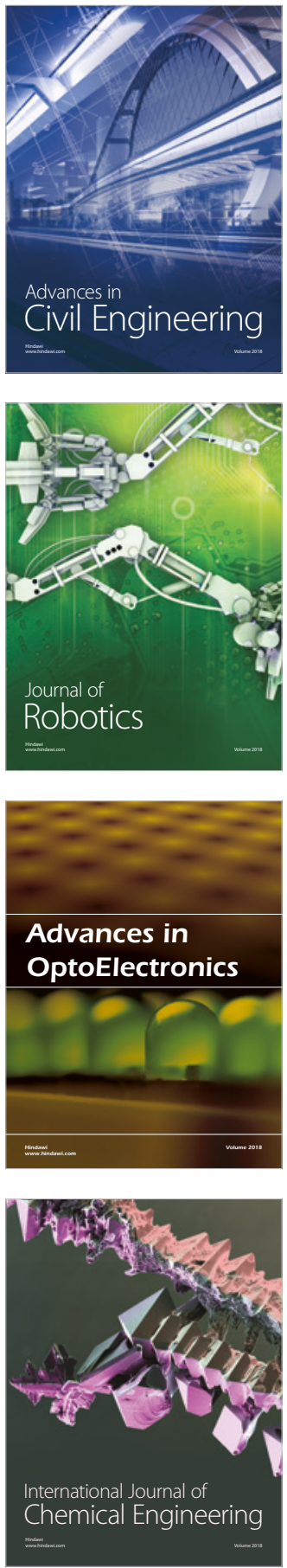

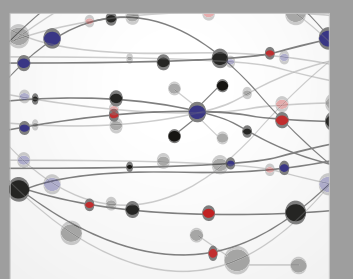

\section{Rotating \\ Machinery}

The Scientific World Journal

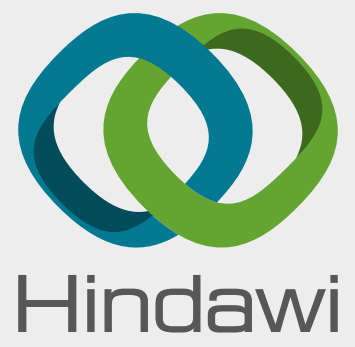

Submit your manuscripts at

www.hindawi.com
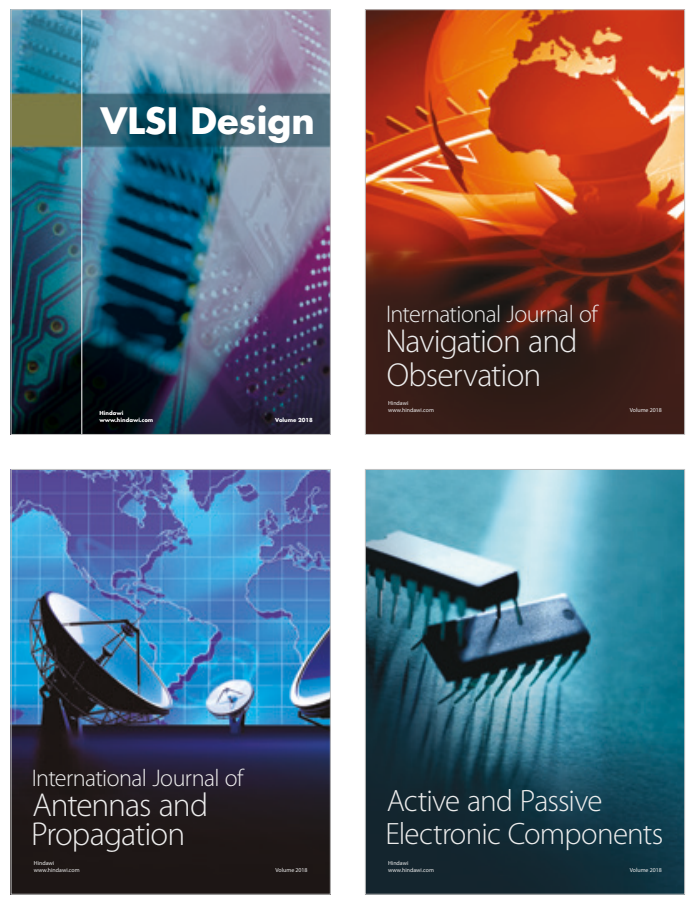
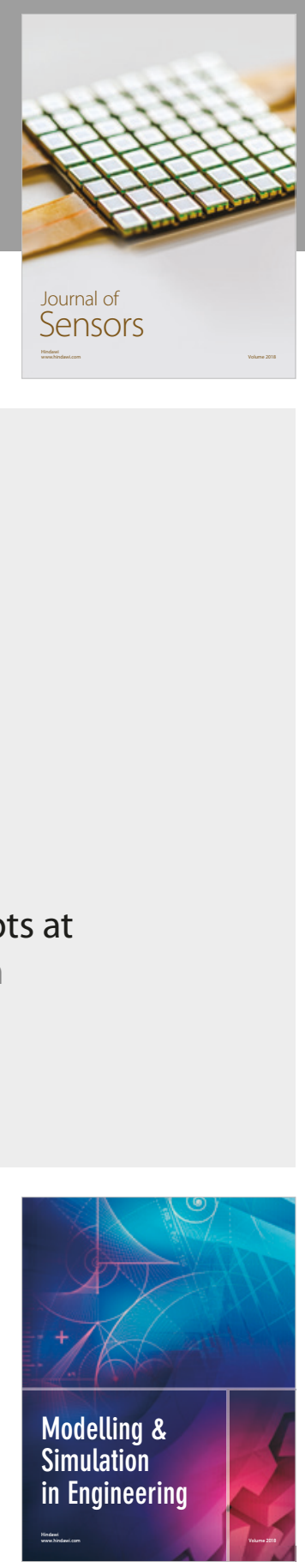

\section{Advances \\ Multimedia}
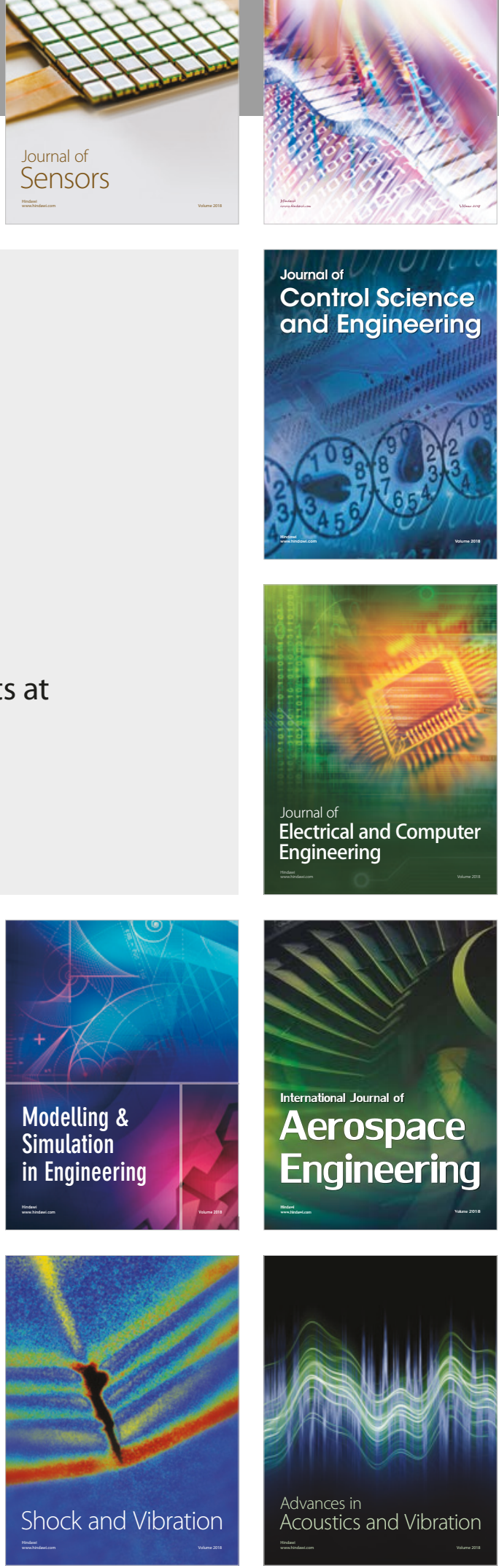